\title{
Evaluation and medical management of the kidney stone patient
}

\author{
Ryan Paterson, MD, FRCSC,; Alfonso Fernandez, MD; ${ }^{\dagger}$ Hassan Razvi, MD, FRCSC; ${ }^{\dagger}$ \\ Roger Sutton, DM, FRCP, FRCPC
}

Can Urol Assoc J 2010;4(6):375-9

\section{Background}

This guideline is structured to provide Canadian urologists with practical recommendations for the evaluation and medical management of kidney stone disease, with a particular emphasis on calcium nephrolithiasis. The goals of this guideline are to promote the identification of significant underlying systemic and urinary abnormalities that would predispose the patient to recurrent nephrolithiasis and to equip urologists with management recommendations that would allow most patients to be treated without referral to other practitioners. This document is not a substitution for a complete review of the literature on all the components of the investigation and management of kidney stone patients. ${ }^{1-41}$

\section{Literature review}

The information included in this document was obtained from a MEDLINE search of the English language literature. Due to the lack of recent randomized controlled trials, references of historical importance are an integral component of these guidelines. Management recommendations are based, where available, on literature published between 2000 and 2010.

The titles of all related articles were reviewed on all key articles. Abstracts were read if the titles were pertinent. English language articles were read if pertinent to the questions to be addressed in the review. In addition, guideline and summary documents produced by other organizations within urology and nephrology were reviewed to maintain consistency in our recommendations.

\section{Kidney stone metabolic evaluation and intervention}

\section{Indications for workup}

\section{Statements}

All kidney stone patients should undergo a limited metabolic evaluation to rule out systemic disease that may present with nephrolithiasis. Level 3 Evidence, Grade C recommendation

Complex patients at potential benefit from detailed metabolic evaluation include (Level 3 Evidence, Grade C recommendation):

- Multiple or bilateral stone disease

- Stones in pregnancy

- Stones in children (<18 years old)

- Non-calcium stones (e.g., cystine, uric acid)

- Recurrent stones

- Strong family history of stones (one or more first-degree relative)

- Stones in setting of a solitary kidney or an anatomical abnormality

- Stones in setting of renal insufficiency

- Systemic disease with increased risk of nephrolithiasis (e.g., gout, bowel disease, distal renal tubular acidosis)

- Critical occupations (airline pilots, sailors, military personnel, firemen)

Due to the low incidence of identified metabolic abnormalities, pure infection stones (e.g., struvite) do not require a complete metabolic evaluation, but close radiological and bacteriological review are recommended. Level 3 Evidence,

\section{Grade C recommendation}

\section{Background}

In a small percentage of patients, nephrolithiasis may be the first presentation of a more serious systemic disease where early identification and treatment can prevent significant complications. Complex patients, due to either potentially serious consequences of a symptomatic stone episode or an increased chance of identifying a specific urinary abnormality requiring tailored treatment, are recommended to undergo a more detailed evaluation. Unfortunately, the literature support for many of these patient groups remains weak. A high interest, even among first-time stone patients, to undergo a detailed evaluation is commonly encountered in clinical practice. The value of empiric recommendations over specific recommendations after a more detailed evaluation is debatable for most uncomplicated stone disease patients. A discussion between the patient and the practitioner regarding compliance with dietary recommendations and compliance with the less commonly required stone- 
Paterson et al.

preventing medications is recommended prior to proceeding with a detailed evaluation in uncomplicated calcium stone disease patients.

\section{Investigations}

\section{Statements}

The history may reveal underlying medical conditions that predispose patients to stone disease, including bowel disease, gout, diabetes and lithogenic medications. Any family history of kidney stones should be elicited (Fig. 1). A dietary history focused on fluid intake and dietary indiscretion will assist in later dietary counselling. Level 1-2 Evidence, Grade

\section{$A-B$ recommendation}

A limited metabolic evaluation for all stone patients will include blood studies (sodium, potassium, chloride, bicarbonate, creatinine, calcium \pm albumin, uric acid and phosphorus), a urinalysis (including urinary $\mathrm{pH}$ ) and a stone analysis. Level 2-3 Evidence, Grade $B-C$ recommendation

Further investigation for hyperparathyroidism, including parathyroid hormone determination, is recommended in the setting of high-normal to elevated serum calcium (in association with calcium phosphate and/or calcium oxalate stones).

\section{Level 2 Evidence, Grade B recommendation}

A detailed metabolic evaluation for complex kidney stone patients (in whom the stones are known or suspected to

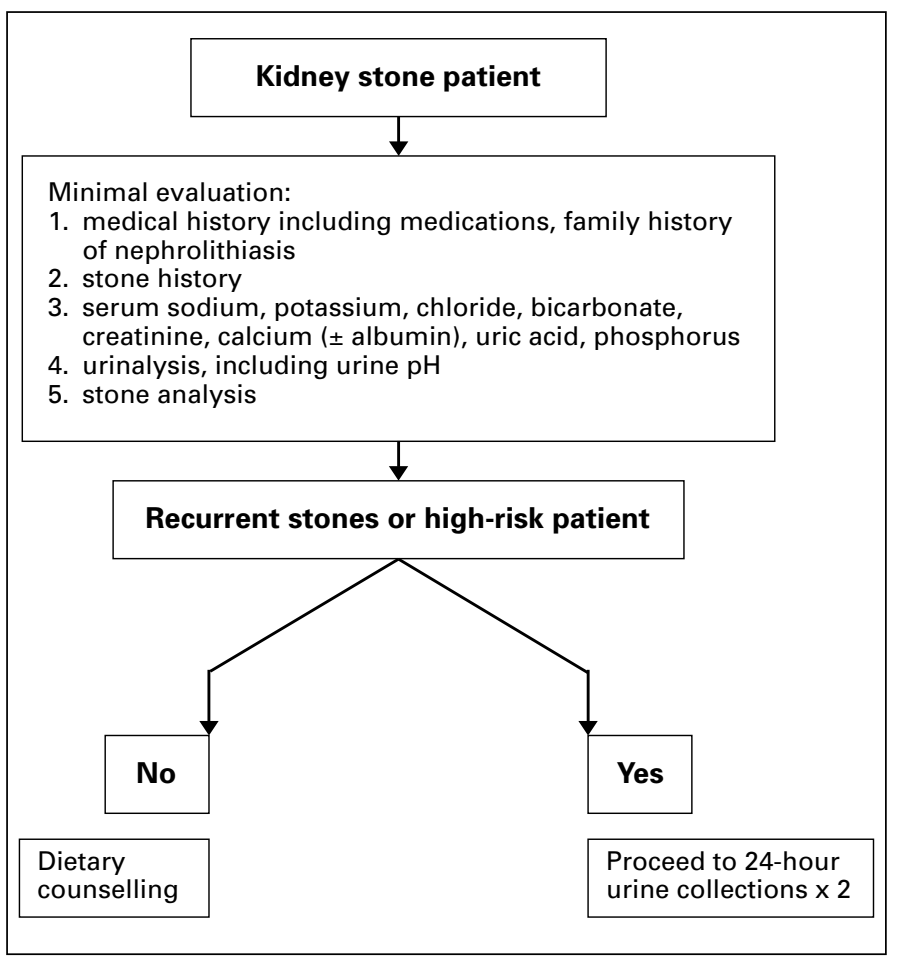

Fig. 1. Algorithm for the initial evaluation of the patient presenting with a kidney stone. be calcium-containing) will include the addition of ideally two 24-hour urine collections (with the patient following their usual diet), including minimum parameters of volume, creatinine, calcium, sodium, citrate, oxalate and uric acid. The collections should occur after the patient has discontinued stone preventing/promoting medications. Level 2-3

\section{Evidence, Grade B-C recommendation}

The primary urinary abnormality in idiopathic uric acid stone formers is excessively acidic $(\mathrm{pH}<5.5)$ urine, not elevated urinary uric acid levels.

A urinary screen for cystine, if the diagnosis of cystinuria is not excluded by stone analysis, should be considered. This commonly consists of a single morning spot urine cystine concentration. Level 4 Evidence, Grade $C$ recommendation

\section{Background}

Stone disease represents a common referral for urologists. To improve the efficiency of stone evaluation, referring physicians could use a questionnaire that includes common stone-predisposing medical conditions, stone preventing/ promoting medications (including vitamins and calcium supplements), family history of stone disease (first-degree relatives), previous stone treatments and prior results of stone disease evaluations. A dietary history, specifically focused on fluid intake as well as consumption of animal protein, dairy products, and salt usage should be included with this questionnaire. Strong evidence that would support going beyond the minimal evaluation in Figure 1 is lacking.

The 24-hour urine collection requires an assessment of the minimum components required of volume, creatinine, calcium, sodium, citrate, oxalate and uric acid. Depending on laboratory availability, additional parameters, such as potassium, phosphorus, sulphate, ammonia and urea nitrogen, can be requested to allow the calculations of supersaturations for calcium oxalate, uric acid and brushite (calcium phosphate). Within the Canadian and European context, the calculation of supersaturation is neither readily available nor required to produce a reduction in stone recurrence.

Screening for primary hyperparathyroidism in the absence of a high-normal or elevated serum calcium is not recommended. The presence of pure calcium phosphate stone disease should prompt a review of the serum electrolytes (to rule out complete distal renal tubular acidosis) and the serum calcium (to exclude primary hyperparathyroidism).

\section{Dietary counselling}

\section{Statements}

All calcium stone formers should be counselled on dietary interventions to reduce stone recurrence or progression. 
Dietary recommendations include (Level 1-3 Evidence, Grade $A-C$ recommendation):

- Increased fluid intake with a goal urine output of $>2$ litres per day

- Reduced salt ingestion ( $<2300$ mg sodium daily)

- Reduced animal protein intake (no more than 2 meals daily with less than 6 to 8 ounces per day)

- Moderate calcium intake (1000 to 1200 mg/day)

- Moderate consumption of high-oxalate content foods (spinach, strawberries, nuts, rhubarb, wheat germ, dark chocolate, cocoa, brewed tea) with limited vitamin C intake to $<1000 \mathrm{mg}$ daily

- Increased intake of citrate-rich fluids (lemonade, orange juice)

Uric acid stone formers may benefit from the above recommendations with emphasis on increased fluid intake and reduced animal protein intake. Level 3-4 Evidence, Grade

\section{$C$ recommendation}

Cystine stone formers will benefit from the above recommendations with emphasis on a substantially increased fluid intake with a goal urine output of $>3$ litres per day. Level 3-4 Evidence, Grade $C$ recommendation

Documented hyperoxaluria should prompt a search for dietary indiscretions, undiagnosed bowel disease or, if substantially elevated, primary hyperoxaluria. Calcium stone formers with idiopathic hyperoxaluria should be treated initially with a low-oxalate diet. Level 2-3 Evidence, Grade

\section{$C$ recommendation}

\section{Background}

Most calcium stone formers can be managed with the empiric recommendations above. An increase in fluid intake to improve urine output remains the strongest single recommendation. Conflicting data exist regarding the value of high citrate-content fluids (lemonade and orange juice) in raising urinary citrate levels where the content of potassium between fluids is discrepant (orange juice rich in potassium may raise urinary citrate levels, while lemonade may have a negligible effect). In addition, large quantities of lemonade or orange juice are required to produce a change in urinary citrate levels with long-term compliance questionable.

A low calcium diet is strongly discouraged in calcium stone formers. Population-based studies, as well as the Borghi study, which compare a low calcium to a normal calcium, low-salt, low-protein diet, support the consumption of the recommended daily allowance (RDA) of calcium set by Health Canada of 1000 to 1200 mg daily. Unfortunately, many Canadians are not meeting the RDA for calcium, increasing the future risk of osteoporosis. Calcium supplements are commonly taken as a substitute or addition to dietary calcium sources. Population-based studies suggest that consumption of calcium supplements should be with meals and not between meals. Due to the prevalence of vitamin $\mathrm{D}$ deficiency in northern climates, moderation, and not avoidance of vitamin D intake, is recommended.

The use of the dietary history during the office evaluation can help with the identification and subsequent alteration of dietary indiscretions. Salt restriction can be difficult due to the prevalence of processed foods in the western diet and an over-reliance on salt for food flavouring. The average sodium intake nationally is $4300 \mathrm{mg}$ per day; Health Canada recommends a daily limit of $2300 \mathrm{mg}$ or one teaspoon. The finding of elevated urinary sodium on detailed evaluation, specifically if combined with concomitant hypercalciuria, should prompt referral to a dietician for more detailed dietary evaluation and counselling. Hypercalciuria, in the setting of elevated urinary sodium, may resolve when patients follow a low-salt diet, which would substantially reduce urinary sodium.

Patients seeking guidance independently (e.g., Internet searches) on stone prevention are confronted with poorly supported recommendations regarding oxalate restriction. Little evidence exists to support a low oxalate diet for the vast majority of calcium stone formers. Further, excessive restrictions of potentially high oxalate content foods may result in a diet suboptimal for cardiovascular health, cancer prevention and glycemic control. Conflicting data exist regarding the effect of vitamin $C$ intake exceeding the RDA and stone formation. Moderation in vitamin $C$ intake is recommended.

Finally, office handouts or office web sites can greatly help patients remember their dietary recommendations.

\section{Medical therapies}

\section{Statement}

Selective management of identified abnormalities from the 24-hour urine collection is recommended (Fig. 2). Level 4

\section{Evidence, Grade C recommendation}

Patients undergoing medical interventions for stone disease should be considered for repeat metabolic assessment (24-hour urine collection), a minimum of 6 to 8 weeks postintervention to assess response to therapy. Level 4 Evidence,

\section{Grade $C$ recommendation}

Patients with hypercalciuria refractory to dietary intervention (particularly moderation of salt intake) should be offered treatment with a thiazide or non-thiazide diuretic (hydrochlorothiazide 12.5 to $25 \mathrm{mg}$ bid; chlorthalidone 12.5 to $50 \mathrm{mg}$ daily; indapamide 1.25 to $2.5 \mathrm{mg}$ daily). Common side effects of these medications include fatigue, dizziness, erectile dysfunction and gout; metabolic changes of glucose intolerance, hypokalemia, hyperuricemia and dyslipidemia can also occur. Level 1 Evidence, Grade $A$ recommendation

An assessment for hypokalemia or (de novo) drug-induced hypocitraturia is required for hypercalciuric stone patients 


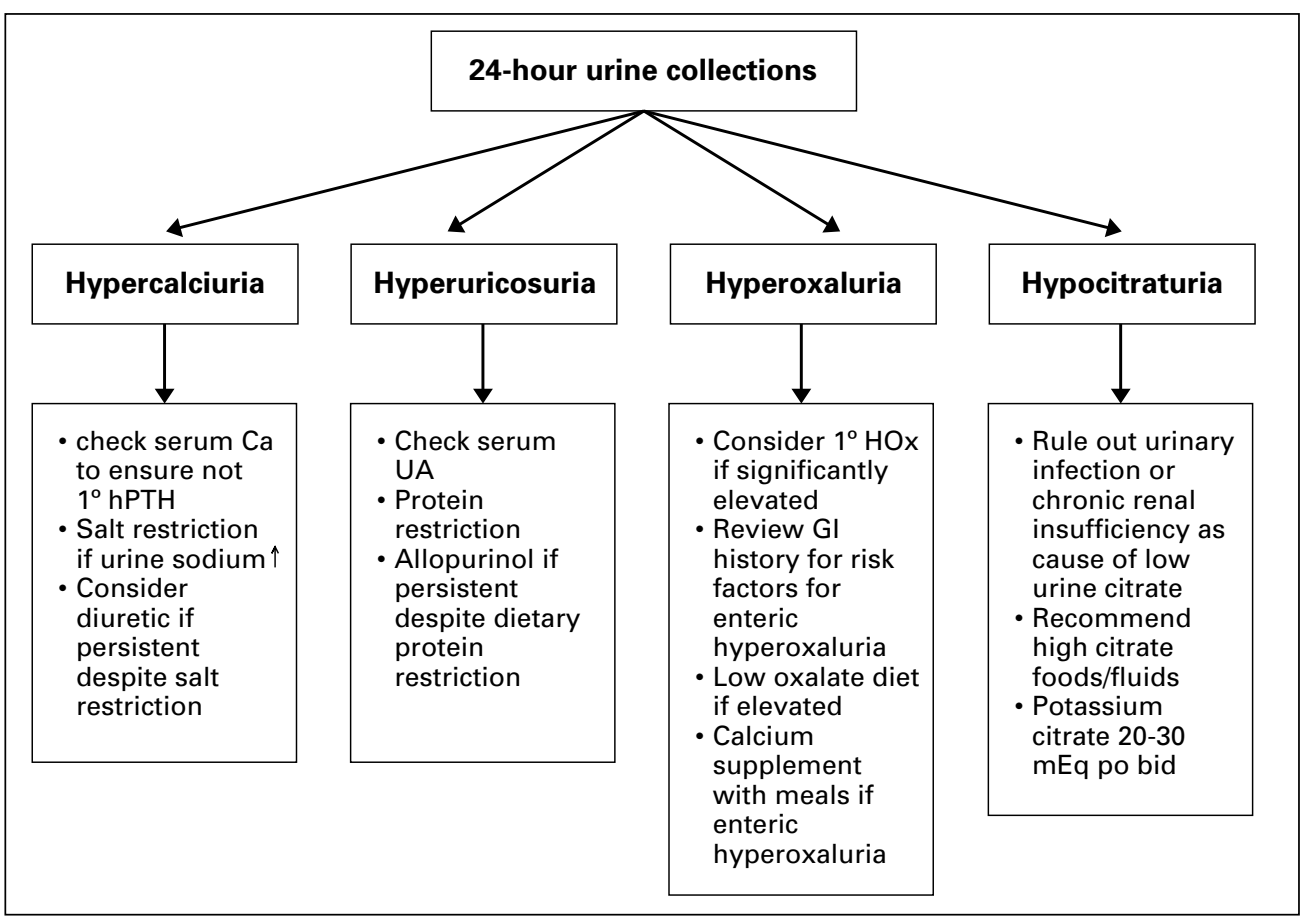

Fig. 2. Algorithm for the 24-hour urine collection. $\mathrm{hPTH}=$ human parathyroid hormone; $\mathrm{UA}=$ urinalysis; $\mathrm{GI}=$ gastrointestinal; $\mathrm{HOx}=$ hyperoxaluria.

once treatment with a thiazide or non-thiazide diuretic is commenced. Hypokalemia and hypocitraturia in this setting are ideally treated with potassium citrate. Level 3 Evidence,

\section{Grade $C$ recommendation}

A multitude of conditions can result in hypocitraturia. Common conditions, such as chronic renal insufficiency and urinary tract infections, must be ruled out in this setting.

Patients with documented hypocitraturia refractory to dietary intervention should be offered treatment with potassium citrate (20 to $30 \mathrm{mEq}$ orally, twice a day). Caution is warranted with use of potassium citrate in the setting of chronic renal insufficiency or concomitantly with other drugs that may limit renal potassium excretion, such as potassium sparing diuretics, angiotensin-converting enzyme inhibitors and angiotensin II receptor antagonists. Level 1-2 Evidence,

\section{Grade $A-B$ recommendation}

Uric acid stone formation can be prevented, and in-situ stones can be dissolved, with urinary alkalinization with monitoring of urinary $\mathrm{pH}$ to ensure a $\mathrm{pH}$ greater than 6.0; potassium citrate is the ideal agent with the alternative of sodium alkali (sodium bicarbonate and sodium citrate). Common side effects of alkalinizing agents include nausea, abdominal bloating and diarrhea. Level 3 Evidence, Grade

\section{$B$ recommendation}

Uric acid stone formers with a history of gout or documented hyperuricosuria refractory to dietary intervention may benefit from treatment with allopurinol (100 to $300 \mathrm{mg}$ daily). Common side effects of allopurinol include liver enzyme abnormalities and rash. Recent investigations suggest a mar- ginal role of hyperuricosuria in promoting calcium nephrolithiasis. Level 3 Evidence, Grade B recommendation

Cystine stone formers require multiple interventions to reduce stone recurrence including dietary measures, urinary alkalinization (to achieve a urinary $\mathrm{pH}$ above 6.5-7.0) and consideration of a thiol-binding agent (D-penicillamine, alpha-mercaptopropionylglycine or captopril). Cystinurics may benefit from multidisciplinary management at a dedicated regional or provincial stone centre. Level 3-4 Evidence, Grade C recommendation

\section{Background}

Selective management involves directed treatment of identified abnormalities from the 24-hour urine collection. Examples include thiazide or non-thiazide diuretics for hypercalciuria refractory to dietary intervention, allopurinol for hyperuricosuria and potassium citrate for hypocitraturia. Non-selective management involves the use of stone preventing medications, such as potassium citrate, to reduce stone recurrence/ progression regardless of any identified urinary abnormality (patient treated often in the absence of a 24-hour urine collection).

Medical management of recurrent kidney stone patients based on the process of correcting specific abnormalities of the 24-hour urine collection(s) will allow most stone patients to be treated by their primary urologist without referral to a dedicated kidney stone centre. Prior to embarking on a course of drug therapy, all patients must be questioned regarding their willingness to comply with lifelong treatment. Once treatment is commenced, response to treatment must be assessed with a repeat 24-hour urine collection, ideally performed a minimum of 6 to 8 weeks after commencing therapy. Further tailoring of therapy can then follow.

Studies evaluating the long-term follow-up of kidney stone patients demonstrate the durability of response to dietary and drug interventions in compliant patients. Unfortunately, loss of patients to follow-up is commonly encountered. The paucity of literature limits the ability to make evidence-based recommendations regarding the timing of follow-up imaging and repeat metabolic evaluation in asymptomatic kidney stone patients. The high recurrence rate of kidney stones provides strength to an argument for 
regular imaging, ideally every 2 to 5 years in asymptomatic patients. Patients with a history of frequent stone recurrences or substantial prior health impairment from prior stone episodes should be considered for more rigorous radiologic follow-up. Asymptomatic renal calculi on surveillance should ideally be imaged on a yearly basis until the images demonstrate a lack of progression. Limiting patient radiation exposure with any imaging follow-up program remains paramount, with reasonable options of a KUB plain film or a renal ultrasound. The recurrence of kidney stones, or the progression of asymptomatic renal calculi on surveillance, should prompt consideration of a repeat metabolic investigation to optimize preventative strategies and to ensure that a more significant metabolic abnormality was not missed during prior evaluation.

"Department of Urologic Sciences, UBC, Vancouver, BC; ' Division of Urologic Surgery, University of Western Ontario, London, ON; 訤ncouver Hospital Stone Centre, Vancouver, BC

Competing interests: None declared.

This paper has been peer-reviewed.

\section{References}

1. Assimos DG, Chew B, Hatch M, et al. Evaluation of the Stone Former. In: Stone Disease $2^{\text {nd }}$ International Consultation on Stone Disease. Paris, France: Health Publications; 2008:33-55.

2. Pearle MS, Coe FL, Rodgers A, et al. Medical Management of Urolithiasis. In: Stone Disease $2^{\text {nd }}$ International Consultation on Stone Disease. Paris, France: Health Publications; 2008:59-84.

3. Taylor EN, Stampfer MJ, Curhan GC. Dietary factors and the risk of incident kidney stones in men: new insights ufter 14 years of followup. J Am Soc Nephrol 2004;15:3225-32.

4. Borghi L, Meschi T, Amato F, et al. Urinary volume, water and recurrences in idiopathic calcium nephrolithiasis:a 5-year randomized prospective study. J Urol 1996;155:839-43.

5. Borghi L, Schianchi T, Meschi T, et al. Comparison of two diets for the prevention of recurrent stones in idiopathic hypercalciuria. N Engl J Med 2002;346:77-84.

6. Borghi L, Meschi T, Guerra A, et al. Randomized prospective study of a nonthiazide diuretic, indapamide, in preventing calcium stone recurrences. J Cardiovasc Pharmacol 1993;22:S78-S86.

7. Wilson DR, Strauss AL, Manuel MA. Comparison of medical treatments for the prevention of recurrent calcium nephrolithiasis. Urol Res 1984;12:39.

8. Ohkawa $M$, Tokunaga $S$, Nakashima $T$, et al. Thiazide treatment for calcium urolithiasis in patients with idiopathic hypercalciuria. Br I Urol 1992;69:571-6.

9. Scholz K, Schwille PO, Sigel A. Double-blind study with thiazide in recurrent calcium nephrolithiasis. J Urol 1982; 128:903-7.

10. Laerum $\mathrm{S}$, Larsen $\mathrm{S}$. Thiazide prophylaxis of urolithiasis. A double-blind study in general practice. Acta Med Scand 1984;215:383-9.

11. Pearle MS, Roehrborn CG, Pak CY. Meta-analysis of randomized trials for medical prevention of calcium oxalate nephrolithiasis. J Endourol 1999;13:679-85

12. Mortensen JT, Schultz A, Ostergaard AH. Thiazides in the prophylactic treatment of recurrent idiopathic kidney stones. Int Urol Nephrol 1986;18:265-9.

13. Barcelo P, Wuhl 0 , Servitge I, et al. Randomized double-blind study of potassium citrate in idiopathic hypocitraturic calcium nephrolithiasis. J Urol 1993;150:1761-4.
14. Pak CY, Sakhaee K, Fuller C. Successful management of uric acid nephrolithiasis with potassium citrate. Kidney Int 1986;30:422-8.

15. Hofbauer J, Hobarth $\mathrm{K}$, Szabo N, et al. Alkali citrate prophylaxis in idiopathic recurrent calcium oxalate urolithiasis- a prospective randomized study. Br I Urol 1994;73:362-5.

16. Mattle D, Hess B. Preventive treatment of nephrolithiasis with alkali citrate— a critical review. Urol Res 2005;33:73-9.

17. Curhan $\mathrm{GC}$, Willett WC, Rimm EB, et al. A prospective study of dietary calcium and other nutrients and the risk of symptomatic kidney stones. N Engl J Med 1993;328:833-8.

18. Curhan GC, Willett WC, Speizer FE, et al. Comparison of dietary calcium with supplemental calcium and other nutrients as factors affecting the risk for kidney stones in women. Ann Intern Med 1997;126:497-504.

19. Curhan $G C$, Willett WC, Knight EL, et al. Dietary factors and the risk of incident kidney stones in younger women: Nurses' Health Study II. Arch Intern Med 2004;164:885-91.

20. Curhan GC, Willett WC, Rimm EB, et al. Family history and risk of kidney stones. J Am Soc Nephrol 1997;8:1568-73.

21. Curhan GC, Willett WC, Rimm EB, et al. Prospective study of beverage use and the risk of kidney stones. Am J Epidemiol 1996;143:240-7.

22. Curhan GC, Willett WC, Speizer FE, et al. Beverage use and risk of kidney stones in women. Ann Intern Med 1998; 128:534-40

23. Curhan GC, Taylor EN. 24-h uric acid excretion and the risk of kidney stones. Kidney Int 2008;73:489-96.

24. Wabner CL, Pak CY. Effect of orange juice consumption on urinary stone risk factors. J Urol 1993; 149:1405-8.

25. Odvina CV. Comparative value of orange juice versus lemonade in reducing stone-forming risk. Clin J Am Soc Nephrol 2006; 1:1269-74.

26. Pak CY, Odvina CV, Pearle MS, et al. Effect of dietary modification on urinary stone risk factors. Kidney Int 2005; 68:2264-73.

27. Pak CY, Peterson R, Poindexter JR. Adequacy of a single stone risk analysis in the medical evaluation of urolithiasis. J Urol 2001;165:378-81.

28. Parks JH, Goldfisher E, Asplin JR, et al. A single 24-hour urine collection is inadequate for the medical evaluation of nephrolithiasis. J Urol 2002:167:1607-12.

29. Yagisawa T, Chandhoke PS, Fan J. Comparison of comprehensive and limited metabolic evaluations in the treatment of patients with recurrent calcium urolithiasis. J Urol 1999;161:1449-52.

30. Ettinger B, Citron JT, Livermore B, et al. Chlorthalidone reduces calcium oxalate calculous recurrence but magnesium hydroxide does not. J Urol 1988;139:679-84.

31. Ettinger B, Tang A, Citron JT, et al. Randomized trial of allopurinol in the prevention of calcium oxalate calculi. N Engl J Med 1986;315:1386-9.

32. Hosking DH, Erickson SB, Van den Berg CJ, et al. The stone clinic effect in patients with idiopathic calcium urolithiasis. J Urol 1983;130:1115-8.

33. Trinchieri A, Mandressi A, Luongo $\mathrm{P}$, et al. The influence of diet on urinary risk factors for stones in healthy subjects and idiopathic renal calcium stone formers. Br I Urol 1991;67:230-6.

34. Goldfarb DS, Coe FL, Asplin JR. Urinary cystine excretion and capacity in patients with cystinuria. Kidney Int 2006;69:1041-7.

35. Lindall A, Denneberg $T$, Edholm E, et al. The effect of sodium intake on cystinuria with and without tiopronin treatment. Nephron 1995;71:407-15.

36. Norman RW, Manette WA. Dietary restriction of sodium as a means of reducing urinary cystine. J Urol 1990; 143:1193-5.

37. Rodman JS, Blackburn P, Williams IJ, et al. The effect of dietary protein on cystine excretion in patients with cystinuria. Clin Nephrol 1984;22:273-8.

38. Nakagawa Y, Asplin JR, Goldfarb DS, et al. Clinical use of cystine supersaturation measurements. J Urol 2000;164:1481-5

39. Brocks $P$, Dahl $C$, Wolf $H$, et al. Do thiazides prevent recurrent idiopathic renal calcium stones? Lancet 1981;2:124-5

40. Mortensen JT, Schultz A, Ostergaard AH. Thiazides in the prophylactic treatment of recurrent idiopathic kidney stones. Int Urol Nephrol 1986;18:265-9.

41. Robertson WG, Peacock M, Selby PL et al. In: Urolithiasis and Related Clinical Research. Plenum Press, New York, NY; 1985:545-8.

Correspondence: Dr. Ryan Paterson, Assistant Professor, Department of Urologic Sciences, UBC, Gordon \& Leslie Diamond Health Care Centre Level 6, 2775 Laurel St., Vancouver, BC V5Z 1M9; fax:604-875-4637; paterson@interchange.ubc.ca 